\title{
A 4-year Follow-up Evaluation Of A Pharmacist-managed Smoking Cessation ProgrammeWith Emphasis On Effectiveness Of Different Treatment Modalities
}

\author{
Phaik Yuan Poh ${ }^{1}$, Celine Chang Chyi $\mathrm{Ng}^{1}$
}

\begin{abstract}
INTRODUCTION Smoking cessation clinic (SCG) was introduced into Singapore's healthcare setting to combat the increase in smoking prevalence. This study aims to determine (1)the effectiveness of a pharmacist-managed SCC, and (2)the association between abstinence rates and different treatment modalities, in Changi General Hospital.

METHODS 445 patients who had attended the SCC were retrospectively reviewed. These patients received structured smoking cessation counselling with or without additional cessation aid comprising of nicotine replacement therapy (NRT), varenicline or bupropion. The study end points were point-prevalence abstinence rates (1)for all subjects, and (2)associated with various treatment modalities, at the final SCC review, 6- and 12-month follow-up.

RESULTS The point-prevalence abstinence rates for all patients at completion of SCG review, 6- and 12-month follow-up were 19.8\%, 23.8\% ( $\mathrm{p}=0.033$ ), and 25.4\% ( $\mathrm{p}=0.009)$, respectively. Abstinence rates between 6- and 12-month follow-up were not statistically different. A significant association between abstinence rates and the use of pharmacotherapy was detected at 6-months' follow-up ( $\mathrm{p}=0.005$ ), but not at SCC review completion or 12-months' follow-up ( $\mathrm{p}>0.05)$. Abstinence rates were highest at $6-$ and 12 -month with use of varenicline (37.4\% and $34.3 \%$, respectively) followed by combination NRT (21.7\% and $26.1 \%)$. Furthermore, patients who had received only smoking cessation counselling had abstinence rates of $20.7 \%$ and $26.0 \%$ at 6- and 12-month follow-up.

concLusions Patient participation in a pharmacist-managed SCC was associated with significantly improved abstinence rates at 6- and 12-months following programme completion. The use of pharmacotherapy was significantly associated with overall abstinence rates at 6-months' followup with varenicline appearing as the most effective smoking cessation aid.
\end{abstract}

AFFILIATION
1 Changi General
Hospital, Singapore
KEYWORDS:
smoking cessation, Pharmacist,
hospital, Counselling,
varenicline, nicotine
replacement
coRRESPONDENCE TO:
Phaik Yuan Poh. Changi General
Hospital, Simei Street 3,
529889 Singapore. Email:
phaikyuan@gmail.com

\section{INTRODUCTION}

Smoking has been a known risk factor for various diseases including lung cancer, chronic obstructive pulmonary disease and also coronary artery diseases ${ }^{1}$. Smoking is reported to be accountable for $87 \%$ and $70 \%$ of lung cancer deaths in men and women respectively ${ }^{2}$. However, it is also the most preventable cause of death in the world. WHO reported that tobacco is currently killing approximately 6 million people each year and by 2030 this figure is expected to increase to more than 8 million people if there are no further measures undertaken ${ }^{3}$.

Despite the reported undesirable consequences of smoking, the Singapore National Survey 2010 showed that the prevalence of adult smoking in Singapore had increased from 13.6\% to $14.3 \%$ between year 2007 to $2010^{1,4}$. Support services for smoking cessation were hence integrated into primary healthcare settings, various non-government organisations and community pharmacy chains.

Pharmacists are readily-available cessation source with the tools and capacity to fight this tobacco epidemic. In the United States of America, a systemic review of 15 studies from year 1980 to 2006 reported statistically significant differences in the effectiveness of smoking cessation services comparing the control groups with those of pharmacist-based intervention ${ }^{5}$. A meta-analysis in year 2008 reported that with the most optimal drugs and counselling, a 1-year abstinence rate of $25 \%$ 
can be expected in smoking cessation ${ }^{6}$. However, there has been a lack of local data to reflect the performance of smoking cessation programme in Singapore.

Based on a previous pilot study that involved 190 patients, the smoking abstinence rate obtained from patients who had attended the Changi General Hospital (CGH) pharmacistmanaged outpatient smoking cessation clinic from September 2008 to July 2010 was examined and the result was comparable to existing published data ${ }^{7}$. While there are many randomised clinical trials conducted in research settings which study the efficacy of different pharmacotherapy in smoking cessation, such efficacy in the real world clinical settings had not been extensively explored in the previous study.

Therefore the aims of this extended study are: (1) To assess the effectiveness of CGH pharmacist-managed smoking cessation clinic from December 2008 till December 2012, and (2)To evaluate the efficacy of different smoking cessation aids prescribed.

\section{METHODS}

\section{Study Design}

This retrospective study includes patients who had attended at least one counselling session of CGH outpatient smoking cessation clinic from December 2008 to December 2012. These include smoking patients who were referred from either inpatient or outpatient by their physicians, and also selfreferred patients. The only exclusion criterion in this study is patients who were under 18 years old. Ethics approval for this project was obtained from the centralised Institutional Review Board of Singapore Health Services (SingHealth).

\section{Details of Smoking Cessation Clinic}

The clinic was run thrice weekly by trained pharmacists certified by the Singapore Health Promotion Board as smoking cessation counsellors. Patients were encouraged to sign up for a package that comprised of three counselling sessions (one first visit up to 45 minutes and two follow-up visits up to 20 minutes) for close monitoring of their smoking status.

\section{Details of Counselling Sessions}

During the first session, patient's medication history, smoking history, previous quit attempts (if applicable), nicotine dependency pattern and motivation determination were assessed. Based on the information gathered, an individualised quit plan was devised. The quit plan comprised of (1)Education on harmful effects of smoking, benefits of quitting and ways to cope with withdrawals symptoms, (2)Recommendation of behavioural modification and non-pharmacological interventions, and (3)Prescription and advice on proper use of appropriate smoking cessation medication (if initiated) based on patient's nicotine dependence and medical history. For those who required a follow up appointment, the date would be recommended by the pharmacist at patient's next availability.

At each visit, measurement and monitoring of breath Carbon Monoxide (CO) levels using Smokerlyzer test was conducted if applicable. If the patient had started on smoking cessation medication, the pharmacist would also review and monitor individual response to drug therapy and document patient's progress in the case notes.

Between visits, telephone surveys were made and patient's treatment progress was assessed. At the final session of the clinic, patients' smoking status was assessed and classified as either no improvement, reduced or stopped smoking. This was chemically verified using the Smokerlyzer test if applicable. Patients with an exhaled CO level of $\leq 3 \mathrm{ppm}$ were considered abstinence from smoking.

\section{Pharmacotherapy}

Under the hospital collaborative practice agreement, pharmacists running the smoking cessation clinic were allowed to prescribe smoking cessation pharmacotherapies that includevarious forms of nicotine replacement therapies (NRTs) such as the gum, lozenge, inhaler and patch, bupropion sustained-release (SR) and varenicline. Patients were counselled on the proper use of the prescribed smoking cessation aid(s)and the choice of aids prescribed was made based on patients' suitability, preference, convenience and also their smoking patterns.

Patients started on pharmacotherapy were closely monitored for side effects via phone call one week after initiation. Pharmacists would advise appropriately based on the severity of adverse reactions reported and document in individual checklist.

\section{Key performance indicators}

After the final clinic session, patients were followed-up at 6- and 12- months via telephone calls to obtain theirselfreported smoking status. Patients were asked if they had smoked any cigarettes in the past 7 days from the day of follow up. Those who self-reported zero sticks would be considered as smoke-free.

Patients who were uncontactable were assumed to be still smoking at those specific time points and were included in statistical analyses. 


\section{Measurable outcomes}

The primary and secondary outcomes of this study were to determine the outpatient immediate abstinence rate, pointprevalence abstinence rate at 6 - and 12 - months and also to assess the comparative abstinence rates between different pharmacotherapies, respectively. In this study, immediate abstinence is defined as abstinence from cigarette smoking on the day of the final clinic session. Point-prevalence abstinence is defined as abstinence from cigarette smoking for at least the past 7 consecutive days from the day of telephone follow-up.

\section{Statistical Analysis}

Descriptive statistical analyses were carried out on the immediate abstinence rates, 6- and 12-month abstinence rates and they were presented as percentages. Multiple McNemar's test (with Bonferroni Adjustment) was used to compare the abstinence rates at completion of final clinic session, 6- and 12-month follow-up. Pearson chi-square test was used to assess the association between abstinence rates and different types of pharmacotherapy, at the completion of final clinic session, 6- and 12-month follow-up. All comparisons were two-sided and a p-value of $<0.05$ was considered statistically significant. Statistical data analyses were performed using SPSS statistical software, version 19.0.

\section{RESULTS}

\section{Baseline demographics}

A total of 445 patients were reviewed in this study and the baseline demographics of these patients are shown in Table 1. Of these patients reviewed, $22.9 \%$ and $26.3 \%$ of patients were lost to follow-up at 6 months and 12 months respectively.

\section{Primary Outcomes}

The overall immediate abstinence rate, point-prevalence abstinence rate at 6-month and 12-month follow-up are shown in Figure 1. At the final smoking cessation clinic review, a total of $19.8 \%$ of patients reported abstinence. This abstinence rate increased to $23.8 \% 6$ months later. The increase continued and recorded a point-prevalence abstinence rate of $25.4 \%$ at 12-month follow up. The difference between the immediate abstinence rate and point-prevalence abstinence rate at both 6- and 12-months was found to be statistically significant with a P-value of 0.033 and 0.009 respectively. However, there was no significant difference detected between the 6- and 12-month point-prevalence abstinence rates.

\section{Secondary Outcomes}

Table 2 demonstrates the abstinence rates obtained from
Table 1. Baseline Demographics of Study Patients.

\begin{tabular}{|c|c|}
\hline Characteristics & Number of Patients $(\%)$ \\
\hline \multicolumn{2}{|c|}{ Gender } \\
\hline Male & $418(93.9)$ \\
\hline Female & $27 \quad(6.1)$ \\
\hline Mean age \pm SD (range) & $\begin{array}{l}50.6 \text { years } \pm 15 \\
\text { (18-92 years) }\end{array}$ \\
\hline \multicolumn{2}{|c|}{ Race } \\
\hline Chinese & $277(62.2)$ \\
\hline Malay & $90 \quad(20.2)$ \\
\hline Indian & $50 \quad(11.2)$ \\
\hline Others & $28 \quad(6.3)$ \\
\hline \multicolumn{2}{|c|}{ Number of sessions attended } \\
\hline 1 & $260(58.4)$ \\
\hline 2 & $88 \quad(19.8)$ \\
\hline 3 & $63 \quad(14.2)$ \\
\hline 4 & $15 \quad(3.4)$ \\
\hline 5 & $8 \quad(1.8)$ \\
\hline 6 & $6 \quad(1.3)$ \\
\hline 7 & $4 \quad(0.9)$ \\
\hline 8 & $0 \quad(0.0)$ \\
\hline 9 & $1 \quad(0.2)$ \\
\hline \multicolumn{2}{|c|}{ Presence of co-morbidities } \\
\hline Cardiovascular & $222(49.9)$ \\
\hline Respiratory & $136(30.6)$ \\
\hline Endocrine & $71 \quad(16.0)$ \\
\hline Psychiatry disorders & $21 \quad(4.7)$ \\
\hline Others & $92 \quad(20.7)$ \\
\hline Nil / Denies & $78 \quad(17.5)$ \\
\hline
\end{tabular}

different treatment modalities used. Among the various treatment options, varenicline consistently achieved the highest abstinence rate throughout the study period. This was followed second by patients who only received behavioural smoking cessation counselling as sole therapy, marking an immediate abstinence rate of $21.3 \%$ while both single NRT and combination NRT only achieved an immediate abstinence rate of $15.0 \%$ and $13.0 \%$ respectively. However, at $6-$ and 12-months follow-up, the point-prevalence abstinence rate achieved with combination NRT increased sharply and overtaken both single NRT and patients who did not receive any pharmacotherapy.

It was shown that there is a significant association between the treatment modalities used and abstinence rates at 6-month follow-up with $\mathrm{P}=0.005$. However, no significant association was detected at smoking cessation clinic completion $(\mathrm{P}=0.101)$ and also at 12-month follow-up $(\mathrm{P}=0.054)$. Treatment 


\section{Research Paper}

Table 2. Abstinence rates achieved with different treatment modalities.

\begin{tabular}{|c|c|c|c|c|}
\hline Treatment modalities & $n$ & $\begin{array}{l}\text { Immediate } \\
\text { abstinence } \\
\text { rate }(\%)\end{array}$ & $\begin{array}{l}\text { 6-month point- } \\
\text { prevalence abstinence } \\
\text { rate }(\%)\end{array}$ & $\begin{array}{l}12 \text { month point- } \\
\text { prevalence abstinence } \\
\text { rate }(\%)\end{array}$ \\
\hline Counselling only & 150 & 21.3 & 20.7 & 26.0 \\
\hline Varenicline & 99 & 26.3 & 37.4 & 34.3 \\
\hline Single NRT & 140 & 15.0 & 18.6 & 18.6 \\
\hline Combination NRT & 46 & 13.0 & 21.7 & 26.1 \\
\hline Bupropion* & 7 & 42.9 & 28.6 & 28.6 \\
\hline NRT and Varenicline* & 2 & 0.0 & 0.0 & 0.0 \\
\hline NRT and Bupropion* & 1 & 0.0 & 0.0 & 0.0 \\
\hline P-value & & 0.101 & 0.005 & 0.054 \\
\hline
\end{tabular}

n Total number of patients in each treatment arm

* Excluded from association analysis because the number of patients was too small

with bupropion, combination of NRT with varenicline and combination of NRT with bupropion were excluded from the association analysis as the number of patients in these treatment arms was too small.

\section{DISCUSSION}

Many studies evaluating the effectiveness of smoking cessation programme were published. These studies reported a 12-month abstinence rate ranging from $14 \%$ to $40 \%^{8-12}$. Our study demonstrated a point-prevalence abstinence rate of $25.4 \%$ at 12 -month follow-up whichis similar to existing published data, including a 12 -month abstinence rate of $25 \%$. This is also consistent with results suggested by Tonnesen where a 1-year abstinence rate of $25 \%$ should be expected from a smoking cessation programme $\mathrm{e}^{6}$.

While most of the studies showed a decrease in abstinence rate over time ${ }^{11-15}$, our study demonstrated a significant increase $(\mathrm{P}<0.05)$ in abstinence rate from clinic completion (19.8\%) to both 6- $(23.8 \%)$ and 12-month $(25.4 \%)$ followup. This is consistent with the results found by Philbrick and colleagues who reported an increase in abstinence rate from $47.6 \%$ to $52.4 \%$ at 3 and 6 months respectively ${ }^{16}$ and also the pilot study performed in CGH previously ${ }^{7}$. The exact reasons contributing to the inconsistencies in results are not clear due to the differences in study designs. However, it is very likely that this is because patients were offered individualised pharmacotherapies based on their preference and suitability in this study instead of randomised treatment as in other clinical trials, hence patientswere more motivated and able to achieve a relatively persistent abstinence throughout.

In year 2004, Zow and colleagues studied the effectiveness of smoking cessation programme in another hospital in
Figure 1. Overall abstinence rate of the smoking cessation clinic at different time points.

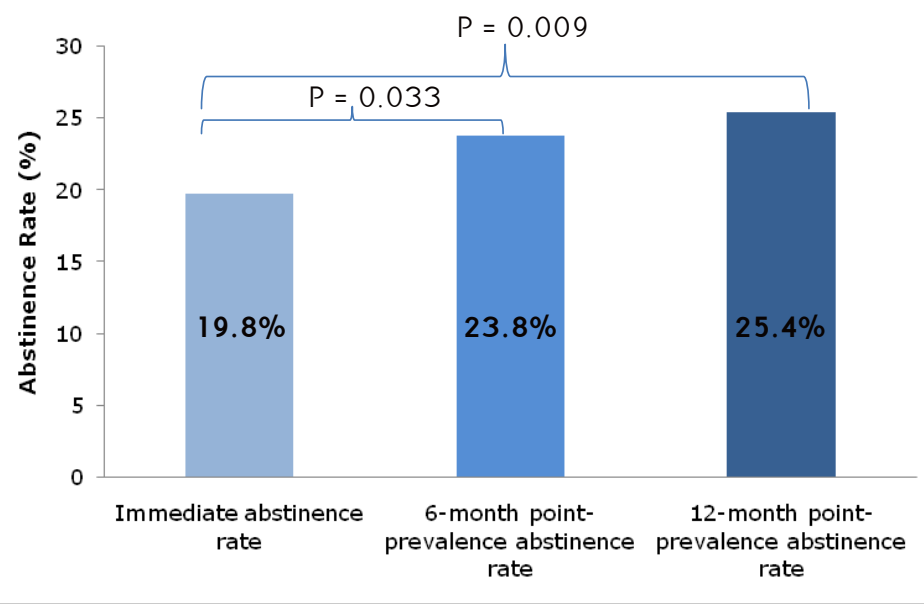

Singapore and found a sustained abstinence rate of $36 \%$ at both 6 and 12 months after final clinic session ${ }^{8}$. As compared to their results, the abstinence rates achieved in this study were lower at all three time-points. One of the possible reasons is that this study has a lower cut-off of the breath $\mathrm{CO}$ level. Patients were considered abstinent at clinic completion if their exhaled CO level was $\leq 3 \mathrm{ppm}$, which is a recommendation from the Singapore Health Promotion Board. However, the cut-off used in the year 2004 study by Zow was $\leq 6 \mathrm{ppm}$.

A meta-analysis suggested that the number of counselling sessions and treatment effectiveness is associated in a doseresponse relationship ${ }^{17}$. This is supported by other studies which found significant increase in abstinence rate in patients who have attended more number of sessions $(\mathrm{P}<0.05)^{8,16}$. Hence, we could consider initiatives to improve the turn up rate of patients as slightly more than half of the patients in this study only attended one counselling session. 
Among the different pharmacotherapies used in smoking cessation, previous studies found varenicline to be superior to bupropion $^{18}$, followed by nicotine replacement therapies ${ }^{19}$. All these aids were shown to be more effective than placebo ${ }^{18-20}$. In general, the results of this study follow the trend observed from previous literature with varenicline alongside with behavioural counselling appearing to be the most effective, with a 12 -month point-prevalence abstinence rate of $34.3 \%$. Although the number of patients in the bupropion arm was small, there is still a promising potential of bupropion in achieving a remarkable point-prevalence abstinence rate of $28.6 \%$ at 12 -month follow-up after varenicline. Thirdly is combination NRT which recorded the lowest immediate abstinence rate of $13.0 \%$, but then increased sharply to $26.1 \%$ after 12 months. Interestingly, the abstinence rates achieved with counselling as the sole therapy were consistently above $20.0 \%$ throughout the study period, marking a 12-month point-prevalence abstinence rate of $26.0 \%$, following very closely after combination NRT. This was then followed by abstinence rate of $18.6 \%$ which was achieved 12 months after treatment with single NRT. This result deviates from published clinical trials which demonstrated NRT to be more effective than placebo ${ }^{16,17,21}$. However, this is consistent with findings obtained by Zow and colleagues whereby the abstinence rates achieved with counselling alone at all time points were higher than those achieved with single NRT $^{8}$. This result might suggest that intensive behavioural counselling can help smokers to achieve abstinence better than NRT in real practice setting. However, as abstinence rate is known to be affected by many variables including level of nicotine dependence, motivation or stress level of the smoker and presence of any psychiatric co-morbidity or misuse of substance ${ }^{17,22}$, further analyses are required to determine the exact factor that might have contributed to the findings of this study.

The association analysis showed that treatment modalities used is significantly associated with abstinence rates only at 6-month follow-up with varenicline recording the highest abstinence rate. This finding might affect the choice of treatment offered to smokers in the future as no significant association between treatment modalities and abstinence rate was detected after 12-month follow-up. Hence, a less costly treatment modality or pharmacotherapy might be favoured especially 6 months after completion of smoking cessation programme to sustain abstinence.

Although this study has minimal exclusion criteria and closely mimics the actual practice setting, there are also several limitations. Firstly is the retrospective nature of the study. Randomisation to treatment arms was not performed and hence the number of participants in certain arms was too small to assess the effectiveness of those treatment modalities. Another limitation is that abstinence at 6- and 12-month follow-up were self-reported by patients and were not validated using any biochemical test. Besides that, all patients who were lost to follow-up were assumed to be still smoking at that specific time point. These might under-report the abstinence rate obtained in this study. Finally, abstinence rates achieved using different smoking cessation aids were not corrected for baseline characteristics of patients such as their Fagerström score of nicotine dependence and previous smoking attempts which all might potentially influence the cessation outcome. Further analysis would be needed to determine the effect of these factors on smoking cessation.

Evidencehasemerged recentlydemonstratingthetolerability and efficacy of combination treatment with varenicline and bupropion in smoking cessation ${ }^{11,23-25}$. However, there is also a study which proposed contradicting result ${ }^{26}$. As this combination treatment is relatively new, none of the patients was treated with this option in this study. Future research is needed in order to determine the efficacy of varenicline and bupropion combination treatment in smoking cessation clinic.

\section{CONCLUSION}

From this study, it can be concluded that the pharmacistmanaged SCC in CGH is able to positively influence smoking cessation. The abstinence rates at 6- and 12-months were associated with a progressive and significant improvement following programme completion and this is comparable with published studies. A significant association was demonstrated between the use of different treatment modalities with overall abstinence rates at 6-months' follow-up with varenicline appearing to be the most effective pharmacotherapeutic agent for smoking cessation. It is also important to recognise that behavioural counselling, as a sole therapy, is able to achieve considerably good abstinence rates throughout the study. Hence, smokers who need assistance to quit smoking are strongly encouraged to attend smoking cessation clinics.

\section{REFERENCES}

1. Epidemiology \& Disease Control Division, Ministry of Health, Singapore. National Health Survey 2010 - Singapore [Internet]. p31-36 Available from: http://www.moh.gov.sg/content/dam/ moh_web/Publications/Reports/2011/NHS2010\%20-\%20low\%20 res.pdf (accessed August 2014)

2. American Cancer Society. Cancer Facts \& Figures 2014. Atlanta: American Cancer Society; 2014. Available from: http://www.cancer. org/acs/groups/content/@research/documents/webcontent/acspc042151.pdf(accessed Sept 2014)

3. World Health Organization. Media centre: Tobacco - Fact sheet No339 [Internet]. Available from: http://www.who.int/mediacentre/ 
factsheets/fs339/en/\#(accessed August 2014)

4. Epidemiology \& Disease Control Division, Ministry of Health, Singapore. National Health Surveillance Survey 2007 - Singapore [Internet]. p15-22 Available from: http://www.moh.gov.sg/ content/dam/moh_web/Publications/Reports/2009/nhss2007. pdf(accessed Sept 2014)

5. Dent LA, Harris KJ, Noonan CW. Tobacco interventions delivered by pharmacists: A summary and systematic review. Pharmacothera py.2007;27(7):1040-51.

6. Tonnesen P. Smoking cessation: How compelling is the evidence? A review. Health Policy. 2009;91Suppl 1:S15-25.

7. Ng YYY, Gwee XL. Effectiveness of an outpatient pharmacistmanaged smoking cessation clinic in a Singapore hospital. Poster presented at: World Tobacco Conference 2012; Singapore.

8. Zow HC, Hsu AAL, Eng PCT. Smoking cessation programme: the Singapore General Hospital experience. Singapore Med J. 2004;45(9):430-4.

9. Kennedy DT, Giles JT, Chang ZG, Small RE, Edwards JH. Results of a smoking cessation clinic in community practice. J Am Pharm Assoc. 2002;42(1):51-6.

10. Maguire TA, McElnay JC, Drummond A. A randomized controlled trial of a smoking cessation intervention based in community pharmacy. Addiction. 2001;96(2):325-31.

11. Ebbert JO, Hatsukami DK, Croghan IT, Schroeder DR, Allen SS, Taylor Hays J, et al. Combination varenicline and bupropion SR for tobacco-dependence treatment in cigarette smokers. JAMA. 2014;311(2):155-63.

12. Jorenby DE, Leischow SJ, Nides MA, Rennard SI, Johnston JA, Hughes AR, et al. A controlled trial of sustained-release bupropion, a nicotine patch, or both for smoking cessation. N Engl J Med. 1999;340(9):685-91.

13. Zillich AJ, Ryan M, Adams A, Yeager B, Farris K. Effectiveness of a pharmacist-based smoking-cessation program and its impact on quality of life. Pharmacotherapy. 2002;22(6):759-65.

14. Roth MT, Westman EC. Use of bupropion SR in a pharmacistmanaged outpatient smoking-cessation program. Pharmacotherapy. 2001;21(5):636-41.

15. Koegelenberg CFN, Noor F, Bateman ED, van Zyl-Smith RN, Bruning A, O'Brien JA, et al. Efficacy of varenicline combined with nicotine replacement therapy vs varenicline alone for smoking cessation. JAMA. 2014;312(2):155-61.

16. Philbrick AM, Newkirk EN, Farris KB, McDanel DL, Horner KE. Effect of a pharmacist managed smoking cessation clinic on quit rates. Pharmacy Practice (Internet) 2009 Jul-Sep;7(3):150-6.

17. Fiore MC, Jaen CR, Baker TB, Bailey WC, Benowitz NL, Curry SJ, et al. Treating tobacco use and dependence: 2008 update. Clinical Practice Guideline. Rockville, MD: U.S Department of Health and Human Services. Public Health Service. May 2008.

18. Jorenby DE, Hays JT, Rigotti NA, Azoulay S, Watsky EJ, Williams $\mathrm{KE}$, et al. Efficacy of varenicline, an a $4 \beta 2$ nicotinic acetylcholine receptor partial agonist, vs placebo or sustained-release bupropion for smoking cessation. A randomized controlled trial. JAMA. 2006;296(1):56-63.

19. Eisenberg MJ, Filion KB, Yavin D, Belisle P, Mottillo S, Joseph L, et al. Pharmacotherapies for smoking cessation: a meta-analysis of randomized controlled trials. CMAJ. 2008;179(2):135-44.

20. Ebbert JO, Hughes JR, West RJ, Rennard SI, Russ C, McRae TD, et al. Effect of varenicline on smoking cessation through smoking reduction: a randomized clinical trial. JAMA. 2015;313(7):687-94.

21. Silagy C, Lancaster T, Stead L, Mant D, Fowler G. Nicotine replacement therapy for smoking cessation (Review). Cochrane
Database Syst Rev. 2004;(3):CD000146.

22. Ho KS, Choi BWC, Chan HCH, Ching KW. Evaluation of biological, psychosocial, and interventional predictors for success of a smoking cessation programme in Hong Kong. Hong Kong Med J. 2016;22(2):158-64.

23. Rose JE, Behm FM. Combination treatment with varenicline and bupropion in an adaptive smoking cessation paradigm. Am J Psychiatry. 2014;171(11):1199-205.

24. Hong A, Elrashidi MY, Schroeder DR, Ebbert JO. Depressive symptoms among patients receiving varenicline and bupropion for smoking cessation. J Subst Abuse Treat. 2015;52:78-81.

25. Vogeler T, McClain C, Evoy KE. Combination bupropion SR and varenicline for smoking cessation: a systematic review. Am J Drug Alcohol Abuse. 2016;42(2):129-39.

26. Shiltz D, Paniagua A, Hastings JE. A retrospective comparison of varenicline monotherapy versus the combination of varenicline and bupropion or bupropion and nicotine patches in a VA tobacco cessation clinic. Journal of Smoking Cessation. 2011;6(1):65-73.
ACKNOWLEDGMENTS

The authors would like to acknowledge Carmen Kam Jia Wen, Research Officer, Clinical Trials and Research Unit, Changi General Hospital, for providing statistical consultation

CONFLICT OF INTEREST

All the authors have completed and submitted the ICME Form for Disclosure of Potential Conflicts of Interest andnone were reported

\section{FUNDING}

There was no source of funding for this research. 